\title{
Rapid formation and flexible expression of memories of subliminal word pairs
}

\author{
Thomas P. Reber ${ }^{1,2}$ and Katharina Henke ${ }^{1,2 *}$ \\ ${ }^{1}$ Division of Experimental Psychology and Neuropsychology, Department of Psychology, University of Bern, Bern, Switzerland \\ ${ }^{2}$ Center for Cognition, Learning, and Memory, University of Bern, Bern, Switzerland
}

\section{Edited by:}

Morten Overgaard, Aalborg

University, Denmark

\section{Reviewed by:}

Giorgio Marchetti, www.mindconsciousness-language.com research net, Italy

Zoltan Dienes, University of Sussex, UK

\section{*Correspondence:}

Katharina Henke, Division of Experimental Psychology and Neuropsychology, Department of Psychology, University of Bern,

Muesmattstrasse 45, 3012 Bern,

Switzerland.

e-mail: henke@psy.unibe.ch
Our daily experiences are incidentally and rapidly encoded as episodic memories. Episodic memories consist of numerous associations (e.g., who gave what to whom where and when) that can be expressed flexibly in new situations. Key features of episodic memory are speed of encoding, its associative nature, and its representational flexibility. Another defining feature of human episodic memory has been consciousness of encoding/retrieval. Here, we show that humans can rapidly form associations between subliminal words and minutes later retrieve these associations even if retrieval words were conceptually related to, but different from encoding words. Because encoding words were presented subliminally, associative encoding, and retrieval were unconscious. Unconscious association formation and retrieval were dependent on a preceding understanding of task principles. We conclude that key computations underlying episodic memory - rapid encoding and flexible expression of associations - can operate outside consciousness.

Keywords: unconscious, non-conscious, implicit, non-declarative, analogical thinking, episodic memory, associative learning, flexibility

\section{INTRODUCTION}

Experiences are incidentally encoded from moment to moment and stored in episodic memory, from where they can be retrieved as a whole or in elements. Episodes consist of perceptual impressions, conceptual aspects as well as temporal and spatial information that are rapidly associated in memory (Sutherland and Rudy, 1989; Eichenbaum et al., 1996; Henke et al., 1997, 1999; O’Reilly and Rudy, 2000; Mayes et al., 2007). Accordingly, episodic memory has been defined as the memory for personal experiences in time and place (Tulving, 1985, 2001). Furthermore, episodic memory is also thought to depend on autonoetic consciousness (Tulving, $1985,2001)$. Autonoetic consciousness refers to a person's awareness of his existence and identity in subjective time extending from the personal past through the present to the future. The term autonoetic refers to the consciousness that allows us to be aware of the subjective time in which events happen. Here, we asked whether consciousness is a necessary condition for rapid associative encoding and the flexible retrieval of the formed associations. We hypothesized that these key computations underlying episodic memory might be operating even in situations where one is unaware of encoding and retrieval.

It is debated whether consciousness is a necessary condition of episodic memory formation (Henke et al., 2003; Degonda et al., 2005; Smith and Squire, 2005; Greene, 2007; Hannula et al., 2007; Henke, 2010). This debate has been partly nourished by findings from studies with subliminal face-word pairs presented for unconscious associative encoding (Henke et al., 2003; Degonda et al., 2005). In these studies, face-word pairs were presented for $17 \mathrm{~ms}$ preceded and followed by pattern masks to avoid stimulus awareness (Henke et al., 2003; Degonda et al., 2005). When participants were later prompted by supraliminal presentations of the encoding faces, their behavior in a forced-choice test indicated that they had associated the subliminal faces to the subliminal words. The newly formed associations between subliminal faces and words were retained over at least $3 \mathrm{~min}$ indicating longterm rather than short-term storage. Because the computations required by this task are reminiscent of episodic memory, we speculated that new episodic memories could be formed and retained even without consciousness of encoding (Henke, 2010). One such computational characteristic that is typical for episodic memory is association formation (McClelland et al., 1995; Henke et al., 1999; O'Reilly and Rudy, 2000, 2001; Norman and O'Reilly, 2003). Our previous studies suggested the feasibility of unconscious association formation (Henke et al., 2003; Degonda et al., 2005). Another computational characteristic that is typical for episodic memory formation and that was operational in our subliminal studies (Henke et al., 2003; Degonda et al., 2005) is one-trial encoding (Squire, 1992; Treves and Rolls, 1994; O’Reilly and Rudy, 2000; Norman and O'Reilly, 2003).

A further characteristic of episodic memories is their compositionality and representational flexibility (Cohen and Eichenbaum, 1993). Compositionality refers to the mental representation of both the entire memory and its individual elements. The elements of episodic memories are not blended into an inseparable representation but are accessible individually and also in relation to each other. The representation of episodic memories is considered flexible because elements and their associations can be reactivated through many routes. Even an aspect of an event that is only remotely related to an encoding episode may reactivate the entire memorized episode. This flexibility allows for a test format that differs from the used study format. Such a representational flexibility also permits the inferential and analogical use 
of episodic memories in new situations (Cohen and Eichenbaum, 1993; Eichenbaum et al., 1996; Mayes et al., 2007).

Our previous experiments on subliminal encoding (Henke et al., 2003; Degonda et al., 2005) had not addressed compositionality and representational flexibility sufficiently. Therefore, we conducted the current experiments. It is generally assumed that only conscious episodic encoding would yield flexible memory representations. Unconscious encoding, however, is generally expected to yield rigid memory representations, in which the elements are blended into inseparable representations. Such rigid representations can only be accessed if the retrieval and encoding situations match (e.g., perceptual priming; Squire, 1992; Cohen and Eichenbaum, 1993; Reber et al., 1996; Mayes et al., 2007). According to more recent views (Reder et al., 2009; Henke, 2010), processing modes and computational characteristics underlying memory formation do not map onto levels of consciousness of encoding. Accordingly, flexible memory representations may also originate from unconscious encoding of information. In the current experiments we tested whether unconsciously encoded word pairs can be reactivated upon confrontation with semantically analogous word pairs. Because encoding and retrieval word pairs were not identical, a successful retrieval would speak to the flexibility of memory representations. To obtain fully unconscious memory representations, encoding word pairs were presented invisible for only $17 \mathrm{~ms}$ flanked by pattern masks (Figure 1; Henke et al., 2003; Degonda et al., 2005).

Because the current experiments are challenging in terms of unconscious computations, we assumed that they would profit from an initial conscious understanding of the task structure. Recent studies have demonstrated that the processing of subliminal stimuli is facilitated by the previous built-up of specific

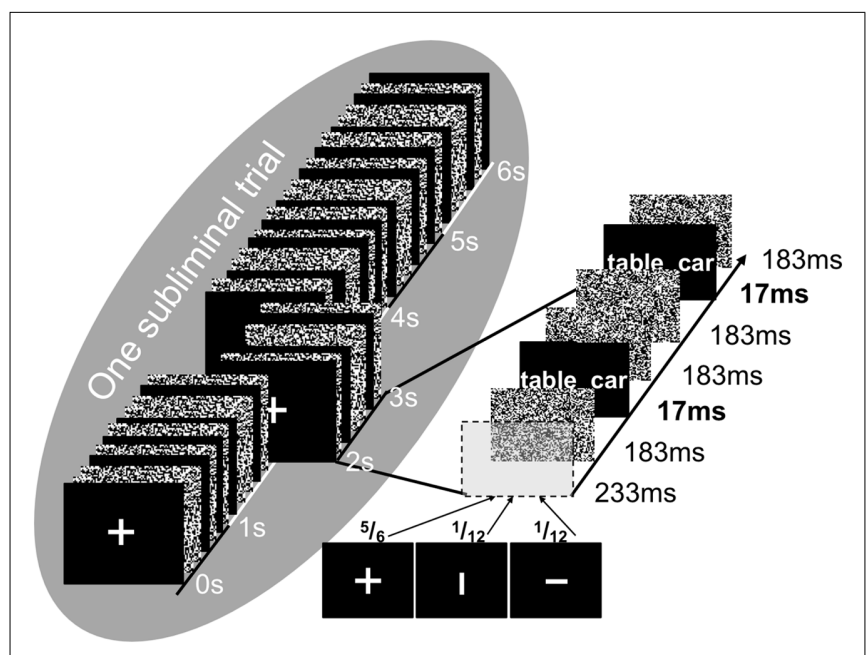

FIGURE 1 | Masking paradigm. Illustrated is one subliminal encoding trial consisting of the 12-fold presentation of the word pair "table-car."

Presentation duration was $17 \mathrm{~ms}$. Word pairs were masked by visual noise patterns. An attention task was included in the masking sequence to ensure that participants visually fixated the center of the screen. For the attention task, participants indicated by button press whether a horizontal or a vertical bar was occurring in place of the fixation cross. The probability of occurrence was $5 / 6$ for a fixation cross and $1 / 6$ for a bar. task expectations and the sensitization of the task-specific processing pathways (Naccache and Dehaene, 2001; Abrams et al., 2002; Kunde et al., 2003; Dehaene et al., 2006; Eckstein and Perrig, 2007; Kiesel et al., 2009; Martens and Kiefer, 2009; Kiefer and Martens, 2010). Therefore, we applied the same memory task (yet with different stimuli) with suprathreshold stimulus presentations for conscious inspection in order to examine potential influences of conscious task understanding on the subsequent unconscious processing of subliminal word pairs. To uncover effects of an initial task understanding in the first experiment, the suprathreshold task version preceded (half of participants) or followed (other half of participants) the task version with subliminal stimulus presentations. Furthermore, we explored whether effects of subliminal processing could be enhanced by repeating the subliminal presentation of a word pair. Accordingly, word pairs in the first experiment were either presented in one or nine subliminal encoding trials.

\section{EXPERIMENT 1}

That the use of analogies requires flexible memory expression can be exemplified by the following tale: The planets orbiting the sun inspired Niels Bohr to formulate his model on how electrons revolve around the atomic nucleus. The detection of analogies between past and current experiences may inspire inferential thinking and problem-solving (Dunbar and Blanchette, 2001). Experiment 1 required a flexible expression of memories because retrieval cues were mere analogs of encoding stimuli. We presented subliminal pairs of unrelated words (e.g., "table-car"; Figure 2) for unconscious encoding. For unconscious retrieval, we presented analogous word pairs (e.g., "desk-bus") with suprathreshold duration allowing the conscious inspection of words. Our aim was to find out whether analogs are sufficient to reactivate originally formed unconscious memories of word pairs. Although the analogs were presented with suprathreshold duration, the hypothesized processes of analogy detection and memory reactivation must run outside consciousness because encoding words were subliminal. To reveal unconscious memory expression, we instructed participants to judge the goodness of the semantic fit between words in an analog (Silberman et al., 2005). In the control condition, the suprathreshold words in a pair were semantically related to two words that each came from a different encoding word pair. When for example the subliminal word pairs "table-car" and "dollar-sheep" were given for encoding, the suprathreshold word pair "counter-goat" was given for retrieval in the control condition (Figure 2). Therefore, we refer to these word pairs as "broken analogs." Words in broken analogs may act as retrieval cues for previously encoded single words but not associations. We assumed that the processing of the subliminal words, like "tablecar," would subjectively decrease the semantic distance between words in analogs, like "desk-bus." Accordingly, we expected a larger number of fit answers to analogs than broken analogs. This result would indicate a flexible expression of unconsciously formed associations.

Experiment 1 contained two subliminal experimental versions: in one experimental version, subliminal word pairs were presented in one encoding trial, and in the other experimental version, subliminal word pairs were presented in nine encoding trials to push effects of unconscious processing. One encoding trial comprised 


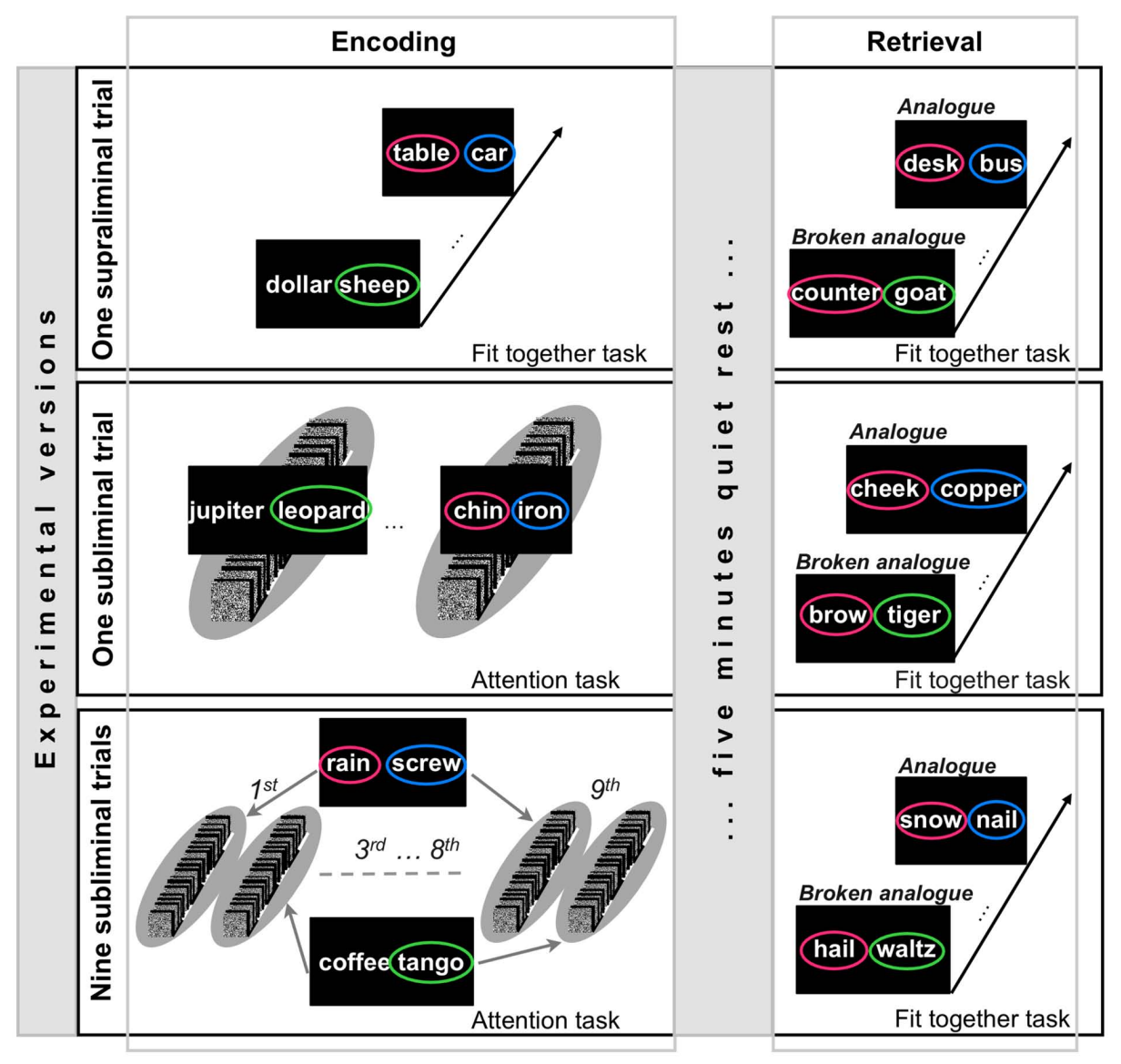

FIGURE 2 | Design of experiment 1. Experiment 1 contained three experimental versions that differed with regard to the encoding mode. Depending on the experimental version, encoding word pairs were presented in one supraliminal encoding trial, in one subliminal encoding trial (illustrated by the gray oval), or in nine subliminal encoding trials. During subliminal presentations, participants performed an attention task. The incidental encoding instruction for suprathreshold word pairs was to indicate whether the two words of a pair fit together semantically or not (Fit together task). In each of the three experimental versions, the encoding run was separated by a 5 -min break from the retrieval run. The design of the retrieval run was identical in the three experimental versions. Retrieval word pairs were presented suprathreshold. The indirect retrieval task required participants to indicate whether the two words of a pair fit together semantically or not (Fit together task).
12 masked 17 ms-presentations of a word pair within a 6-s time window (Figure 1; Henke et al., 2003; Degonda et al., 2005).

Furthermore, a suprathreshold version of the experiment was applied to participants either preceding the two subliminal experimental versions or not. This manipulation should reveal whether an initial versus later conscious apprehension of task principles would facilitate the ensuing unconscious processing of subliminal word pairs (Martens and Kiefer, 2009; Kiefer and Martens, 2010). In the suprathreshold version of the experiment, both encoding and retrieval word pairs were presented for conscious inspection. Importantly, words presented in the suprathreshold version differed from words presented in the subliminal versions of the experiment. Hence, supraliminal words and word pairs could not prime subliminal words and word pairs.

\section{MATERIALS AND METHODS Participants}

Thirty-five healthy students ( 18 women and 17 men; age range: $18-30$ years; $M=25.02, \mathrm{SD}=2.93$ ) participated for remuneration or course credit. They were normally sighted, native German speakers, reported no current or past neurological or psychiatric disorders, and denied taking prescription or illegal drugs. Participants were kept naïve as to the purpose of the experiment (subliminal perception and memory). They were misinformed that the subliminal experimental versions were about attention (encoding part) and language (retrieval part). The local ethics committee approved the study.

\section{Apparatus and materials}

Stimuli were presented with an LCD video beamer with a refresh rate of $60 \mathrm{~Hz}$. The stimulated visual field was $17^{\circ}$ wide and $11^{\circ}$ high. Stimuli consisted of 96 triplets of subordinate terms that belong to the same superordinate category (e.g., table, desk, and counter). We combined words from different categories to form word pairs for encoding. These encoding words were not presented for retrieval. Instead, retrieval words used in the experimental condition were semantic neighbors of words combined in an encoding pair (encoding: table-car; retrieval: desk-bus). We refer 
to the retrieval word pairs used in the experimental condition as "analogs." Retrieval words used in the control condition were semantic neighbors of words presented in two different encoding word pairs (encoding: table-car... dollar-sheep; retrieval: counter-goat; Silberman et al., 2005). Therefore, we refer to the retrieval word pairs presented in the control condition as "broken analogs." We needed the 96 triplets (three versions of a concept) to construct three lists of 16 word-word combinations. Three versions of a concept were needed because each list contained 16 encoding word pairs, 16 analogs, and 16 broken analogs. The three lists were used for the three versions of the experiment (see below "Design and Procedure").

To distribute effects of a potential stimulus bias over conditions, we counterbalanced stimuli across conditions: encoding word pairs were rearranged such that analogs appeared as broken analogs and vice versa. We included a baseline condition in anticipation of later experimentation with neuroimaging. For this baseline condition, we created 96 pairs of consonant strings (e.g., brtmzh-nvpthw), of which 16 pairs were used in the encoding part and 16 in the retrieval part of each of the three experimental versions. Sixty further words were used as targets and distracters in a direct test of conscious awareness of subliminal stimuli. This awareness test was carried out at the end of the session.

\section{Masking paradigm}

We used the masking technique of Degonda et al. (2005) to present the encoding word pairs subliminally. A subliminal word pair was flashed 12 times for $17 \mathrm{~ms}$ during a subliminal trial of $6 \mathrm{~s}$. The 17-ms-presentations were flanked by pattern masks to avoid the visibility of the word pair. Masks consisted of random patterns of black and white pixels $(800 \times 600)$. During subliminal trials, participants performed an attention task that ensured that their attention was focused and maintained. For this attention task, we presented fixation crosses, horizontal and vertical bars intermixed with word pairs and masks embedded in a 6-s subliminal trial (Figure 1). A fixation cross or a bar was presented once per second in all subliminal trials. Once in 12 presentations or within one subliminal trial, the fixation cross was randomly replaced by a vertical or horizontal bar. Participants' task was to indicate the bar's orientation by button press. The left-hand word of a subliminal word pair appeared to the left of a subsequently or previously flashed fixation cross/bar and the right-hand word appeared to its right, which constitutes a bilateral simultaneous hemispheric stimulation. The presentation durations were $17 \mathrm{~ms}$ for stimuli $(S), 183 \mathrm{~ms}$ for masks $(M)$, and $233 \mathrm{~ms}$ for fixation crosses/bars $(F)$. One subliminal trial (Figure 1) consisted of the following image sequence: $F-M-S-M-M-S-M-F-M-S-M-M-S-M-F-$ $M-S-M-M-S-M-F-M-S-M-M-S-M-F-M-S-M-M-S-M-F-M-$ $S-M-M-S-M$. To conceal the purpose of the masking sequence, participants were told that the masking sequence measured attention alone.

\section{Design and procedure}

Participants were seated in the darkened laboratory and took a health questionnaire at the beginning of the session. Their heads were positioned on a chin rest. Before the experiment started, they underwent practice trials to get familiar with the tasks and procedures. There were three versions of the experiment. Word pairs were subliminal in two versions and suprathreshold in one version. Each participant took all three versions of the experiment. The order in which experimental versions were given to participants was varied between participants. Half the participants took the suprathreshold version before the two subliminal versions. This order was reversed for the other half of participants. We hypothesized that the task structure would become apparent to participants and would facilitate the ensuing processing of the subliminal word pairs, if the suprathreshold version was taken at the beginning of the session.

Each experimental version consisted of an encoding part, followed by 5 min of quiet rest. Then, a retrieval part followed. In the suprathreshold version, the 16 encoding word pairs were presented above threshold to assess the incidental encoding of consciously apprehended word pairs. In one suprathreshold encoding trial, one word pair (e.g., table-car) was shown for $3.5 \mathrm{~s}$, followed by a 1-s inter-stimulus interval. The encoding task was to judge the semantic relatedness of the two words in an encoding pair (yes or no) immediately upon its occurrence. Participants were instructed in the following way: "You will be presented with a sequence of word pairs. Your task is to decide whether the two words in a pair fit together or not regarding their meaning. If you think that the two words in a pair fit together, push the left mouse button. If you think the two words do not fit together, push the right mouse button. [...] The words in a pair may not fit together at first glance. But there may be more remote associations between the two words. Really close associations as present in "needle-yarn" will not occur. An example word pair is "cow-grill." Although cow appears not associated to grill, cow and grill may still be judged as fitting together because beef is a popular meat used for barbecues. For some words in pairs you will find associations easily. Other words may be more remotely associated. Please try to establish a criterion for yourself that allows you to give an approximately equal number of fit and don't fit answers." The criterion for deciding about the semantic fit between words was established during the practice trials given at the beginning of the session. In the baseline condition, where consonant strings were presented, participants judged the visual relatedness of the two consonant strings in a pair (yes or no). For both conditions (i.e., for semantic and visual relatedness judgments), we asked participants to use a loose response criterion in order to approximate $50 \%$ fit answers. In the two subliminal experimental versions, encoding word pairs were either presented in one subliminal encoding trial (12 masked presentations of the same word pair in a 6-s time window, Figure 1), or in nine temporally dispersed subliminal encoding trials (nine times a 6-s time window entailing 12 masked presentations of the same word pair). The nine subliminal encoding trials of a given word pair (e.g., "rain-screw," Figure 2) were evenly distributed over the whole encoding run. Hence, the nine subliminal encoding trials of a word pair were intervened by subliminal presentations of other word pairs (e.g., "coffee-tango") used in the condition and by subliminal presentations of pairs of letter strings used in the baseline condition (Figure 2). Only encoding word pairs, but not the pairs of letter strings, were presented nine times. Consequently, the encoding runs of the three experimental versions had different lengths: the suprathreshold encoding run lasted $2.4 \mathrm{~min}$, 
the subliminal encoding run that included one encoding trial per word pair lasted $3.2 \mathrm{~min}$, and the subliminal encoding run that included nine encoding trials per word pair lasted $16 \mathrm{~min}$. To alleviate tiring, the 16-min run was subdivided into three parts with 1 min breaks in between.

The retrieval parts were the same for all three experimental versions. Retrieval word pairs were presented above threshold for $3.5 \mathrm{~s}$, followed by a $1-\mathrm{s}$ inter-stimulus interval. The indirect retrieval task was the same task as given for conscious encoding, namely to judge the semantic relatedness of words in a pair (yes or no). In the baseline condition, where consonant strings were presented, participants judged the visual relatedness of the two strings in a pair (yes or no).

For encoding and retrieval, word pairs and letter strings were presented in separate blocks of four stimuli, arranged by condition. Hence, the 16 stimuli of each condition were presented in four blocks of four stimuli. Stimulus pairs were randomly assigned to blocks and this assignment differed between participants. For the experimental version with nine encoding trials per word pair, word pairs were randomly assigned to the four blocks of the initial presentation and then reassigned randomly to the following four blocks for each of the following repetitions. The order of blocks was varied between participants to balance sequence effects. We counterbalanced stimuli across conditions.

\section{Awareness test}

Following the above experiment, participants were interviewed on whether they had suspected or even perceived subliminal stimuli or perceptual fragments thereof (see Post-Experimental Interview in Appendix). Then, they were informed about the fact that there were subliminal stimuli embedded in the attention task. Finally, participants underwent the awareness test, where stimulus discriminability was assessed objectively.

Standard procedure to establish a claim of unconscious processing is to demonstrate effects of subliminal stimuli in indirect tests (as used in the experiment) in the absence of significant discriminability of subliminal stimuli in an intentional encoding and direct retrieval test (Cheesman and Merikle, 1984). Our direct test of awareness consisted of 30 trials. In each trial, we presented one subliminal word at the same screen location where the right-hand word in the word pairs presented in the experiment had appeared. Thus, due to participants' central visual fixation, we stimulated their left (language-dominant) hemisphere. The reason why we presented one instead of two words (as in the experiment) was to facilitate subliminal processing by reducing the amount of simultaneously displayed information and by getting rid of the effect of inter-hemispheric inhibition on the simultaneous word processing within each hemisphere (Cook, 1986). We figured that conscious awareness of subliminal word pairs could be safely excluded in the experiment if even single words could not be discerned in the final awareness test. Apart from presenting only one instead of two words, the presentation procedure for a subliminal stimulus was the same as in the experiment: each word was presented in one subliminal trial - i.e., in 12 flashes of $17 \mathrm{~ms}$ each, flanked by pattern masks and intermixed with fixation crosses or bars for the attention task. Immediately following the presentation of one word in one subliminal trial, participants chose between a target and a distracter word (forced-choice test). The target word was the word presented in the subliminal trial. The distracter word was a new word that was unrelated to the target. Participants were instructed to select the word that they thought was subliminally presented. Our standard for claiming the absence of conscious perception of subliminal words was a non-significant $(p>0.200)$ group analysis ( $t$-test) of number of correct choices per participant. Even if this group analysis yielded a non-significant result, we still excluded participants from the analysis of the data from the experiment, if they had reached more than 20 out of potentially 30 correct choices in this awareness test. Twenty correct choices corresponded to the 5\% cut-off of the chance distribution of correct choices. Moreover, we regressed the discrimination performance achieved in the awareness test onto the performance achieved in the indirect retrieval test applied in the experiment to see whether unconscious processing persists even if potential contributions by conscious stimulus discrimination are regressed out (Greenwald et al., 1995).

\section{RESULTS AND DISCUSSION}

No participant reported to have suspected or perceived subliminal stimuli during the experiment. The direct test of stimulus awareness indicated a chance group performance, $M_{\text {correct }}=48.5 \%$, $\mathrm{SD}_{\text {correct }}=9.2 \% ; t(34)=-0.977, p=0.336, r^{2}=0.026$, and no participant reached more than 20 correct choices $(=5 \%$-cut-off of the chance distribution) in the forced-choice test.

The mean number of correct answers given in the attention task during the masking sequence of the experiment was high $(M=94.9 \%$; $\mathrm{SD}=5.8 \%)$. This high accuracy indicates that participants had visually fixated the screen center and were closely attending to subliminal stimulations.

In the experimental version with suprathreshold encoding pairs, participants gave more fit answers to analogs $(M=47.9 \%$, $\mathrm{SD}=14.6 \%)$ than broken analogs $(M=41.4 \%, \mathrm{SD}=12.3 \%)$, $t(34)=2.29, p=0.029, r^{2}=0.133$. This result suggests that conceptual associations had been established between the words during incidental encoding and biased the subjective sense of the semantic relatedness of words contained in the analogs given for retrieval. Hence, the encoded associations between words must have been semantic and were expressed flexibly in the new retrieval context.

The same result occurred for the two subliminal versions of the experiment. We computed a repeated-measures analysis of variance (ANOVA) with the within-subjects factors Encoding Intensity (one versus nine subliminal encoding trials) and Retrieval Condition (analogs versus broken analogs), and with the betweensubjects factor Sequence (suprathreshold version first versus subliminal versions first). The dependent variable was the percentage of fit answers. The interaction of Sequence $\times$ Retrieval Condition was significant, $F(1,33)=5.57, p=0.024, \eta_{p}^{2}=0.171$ while the main effect of Retrieval Condition was not, $F(1,33)=2.49$, $p=0.124, \eta_{p}^{2}=0.083$. Post hoc $t$-tests revealed that analogs received more fit answers $(M=42.5 \%, \mathrm{SD}=8.5 \%)$ than broken analogs $(M=35.5 \%, \mathrm{SD}=9.5 \%), t(16)=2.68, p=0.016$, $r^{2}=0.310$, if the suprathreshold version preceded the two subliminal versions of the experiment (Figure 3A). If the session started with a subliminal version, participants gave a statistically equal 

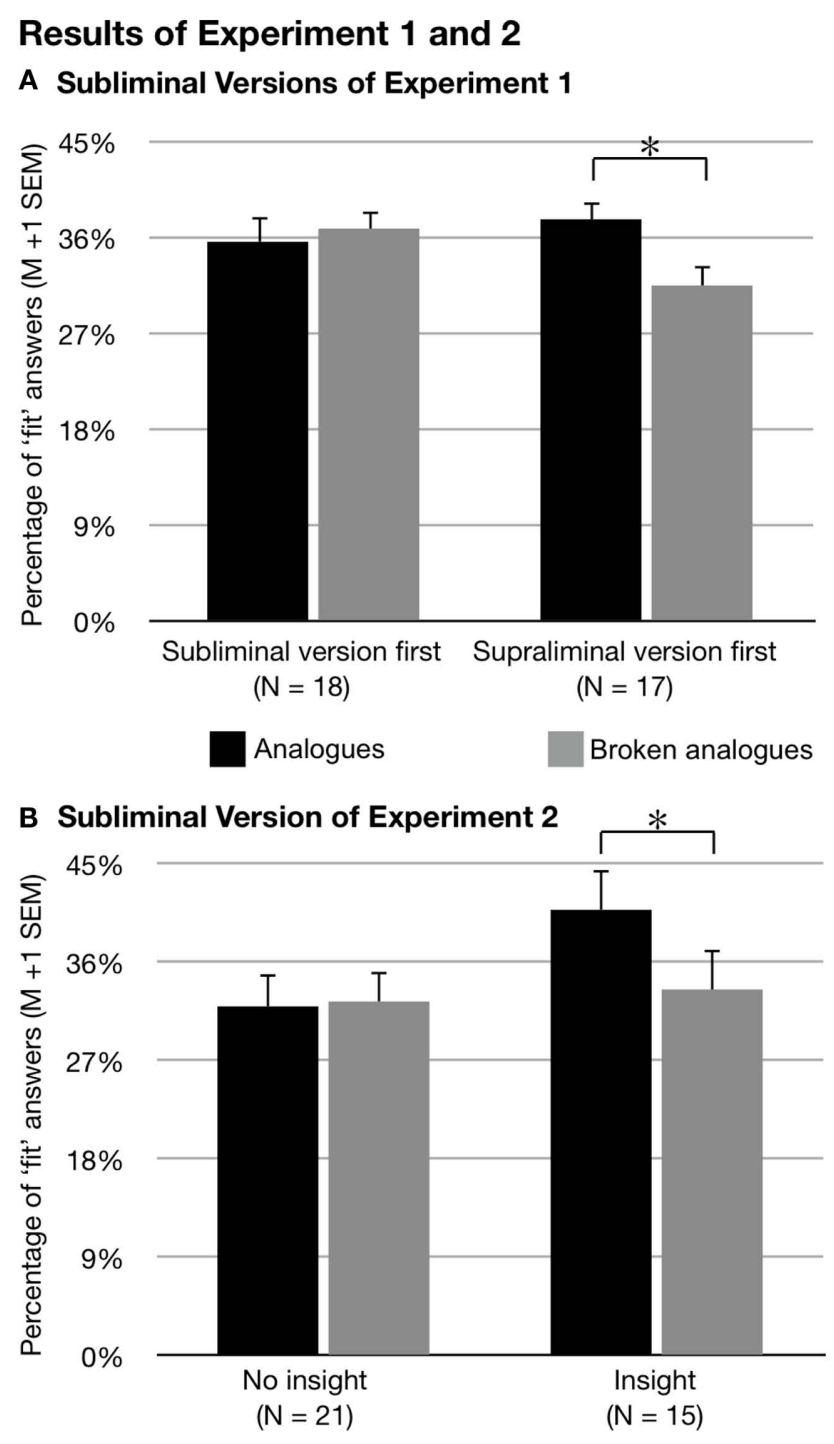

FIGURE 3 | Results of experiment 1 and 2. In both experiments, participants gave more fit answers to analogs than broken analogs following unconscious paired-associative encoding of subliminal word pairs. (A) This effect occurred if participants acquired a task-set in an initial run with suprathreshold presentations of encoding stimuli. (B) An important aspect of acquiring the relevant task-set was gaining insight into the conceptual relation between encoding word pairs and retrieval word pairs. $M$, mean; SEM, standard error of the mean.

percentage of fit answers to analogs $(M=40.1 \%, \mathrm{SD}=12.1 \%)$ and broken analogs $(M=41.5 \%, \mathrm{SD}=8.1 \%), t(17)=-0.57$, $p=0.572, r^{2}=0.018$. The suprathreshold experimental version had apparently informed participants of the task structure and configured their expectations and cognitive processes for the subliminal task. We refer to this configuration of cognitive processes as an installation of a task-set (Monsell, 2003). Because the same kind of cognitive processing is required by the suprathreshold and the subliminal versions of the experiment, the task-set that was already installed during the suprathreshold experiment was maintained and benefited the performance in the subliminal experimental versions. Importantly, different sets of words were used for the three experimental versions. Therefore, the effect of the initial supraliminal experimental version cannot reflect word priming.

The non-significant interaction of Encoding Intensity with Retrieval Condition, $F(1,33)=0.12, p=0.728 \eta_{p}^{2}=0.002$, indicated that the ninefold and one-time subliminal encoding were equivalent in terms of unconscious retrieval performance. The percentage of fit answers was comparable for analogs presented in the experimental version with one encoding trial $(M=43.4 \%$, $\mathrm{SD}=10.6 \%)$ and nine encoding trials $(M=44.3 \%, \mathrm{SD}=13.2 \%)$. Also the percentage of fit answers to broken analogs was comparable between the experimental version with one encoding trial $(M=41.1 \%, \mathrm{SD}=11.94 \%)$ and nine encoding trials $(M=41.4 \%$, $\mathrm{SD}=13.0 \%)$.

A further method (Greenwald et al., 1995) to establish claims of unconscious processing entails the regression of the discrimination performance achieved in the direct awareness test (percentage of correct minus percentage of incorrect answers) onto the performance achieved in the indirect retrieval test applied in the experiment (percentage of fit answers given to analogs minus percentage of fit answers given to broken analogs during the subliminal versions of the experiment). A claim of unconscious processing is considered justified if the $y$-axis intercept is significantly different from zero. This result indicates that unconscious processing persists even if potential contributions by conscious stimulus discrimination are regressed out. We computed a regression analysis for the group of participants who had started with the suprathreshold version of the experiment and who showed an effect of subliminal stimulus processing. The $y$-axis intercept $(0.057, \mathrm{SE}=0.021)$ was significantly greater than zero $[t(16)=2.668, p=0.018]$. This indicates that effects of subliminal perception persisted even if potential contributions of conscious stimulus discrimination were excluded. The slope $(B=-0.235, \mathrm{SE}=0.101, \beta=-0.514)$ was also significant showing an inverse relationship $[t(16)=-2.323, p=0.035]$. The slope of the regression and the distribution of participants above and below the zero line of the $x$-axis (Figure 4A) indicate that the worse stimulus discriminability was in the awareness test, the better was implicit retrieval performance in the experiment. If anything, conscious stimulus discriminability in the experiment might have counteracted unconscious paired-associative encoding and retrieval.

\section{EXPERIMENT 2}

The results of the first experiment suggested that the flexible expression of unconsciously acquired memories can be achieved, if participants are given the chance to get familiar with the task structure during an initial suprathreshold version of the task. The second experiment was conducted to find out whether a critical aspect of the beneficial effect of the initial suprathreshold run is the awareness of the conceptual connection between study word pairs (e.g., "table-car") and test word pairs (e.g., "desk-bus"). Furthermore, we were interested in replicating the finding that a single subliminal encoding trial was sufficient to bias the subjective sense of semantic relatedness of words in analogs presented at retrieval. 

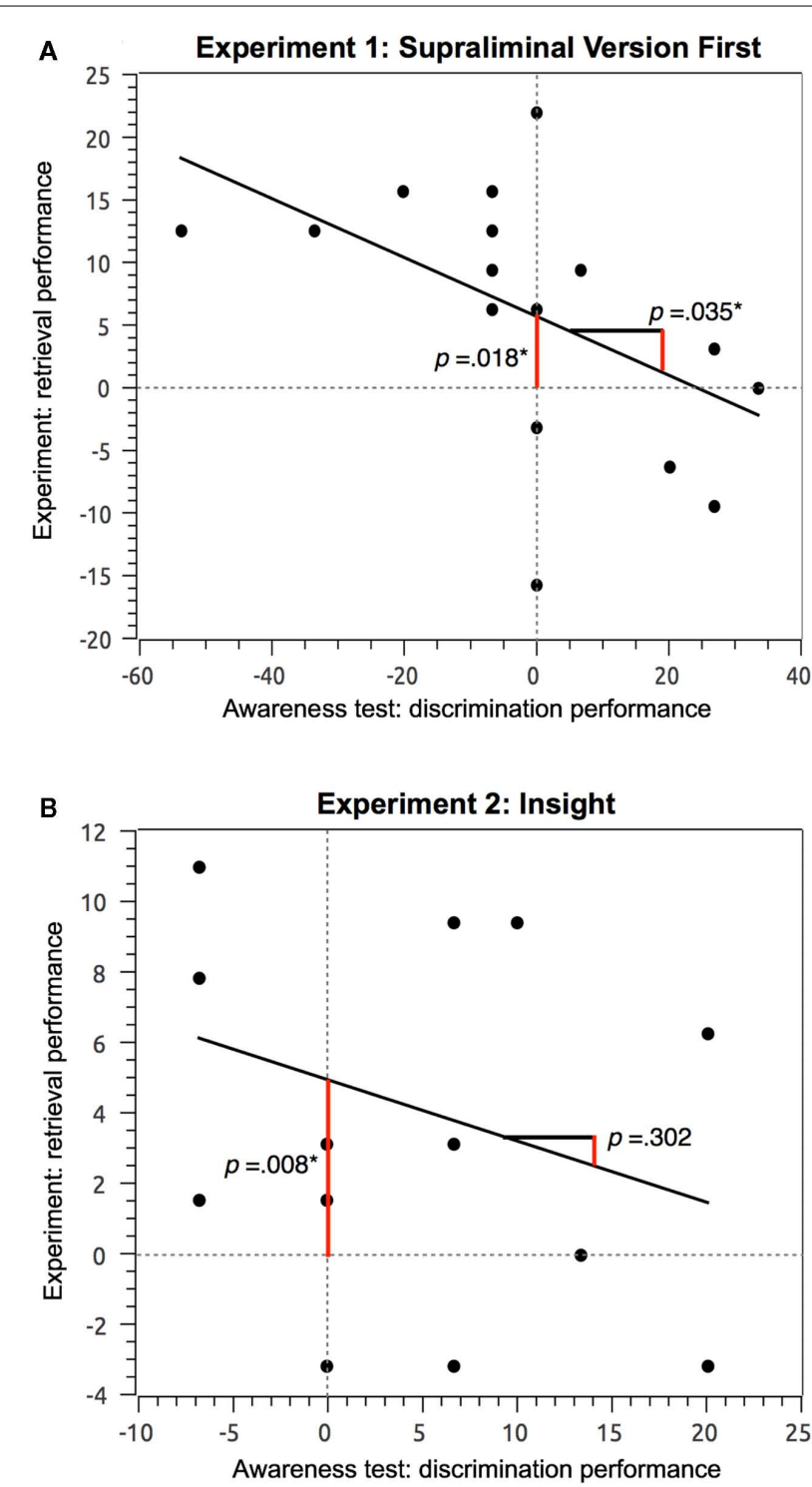

FIGURE 4 | Regression of awareness test performance onto retrieval performance. The measure of discrimination performance was the difference between the percentage of correct answers and the percentage of incorrect answers given in the awareness test. The measure of retrieval performance in the experiments was the difference between the percentage of "fit" answers given to analogs versus broken analogs. (A) Experiment 1. Discrimination performance was regressed onto retrieval performance using the data from participants who took the suprathreshold version first. The significant size of the $y$-axis intercept indicates that the retrieval effect persists when potential contributions of conscious stimulus discrimination are excluded. The slope of the regression was also significant showing an inverse relationship. The slope and the distribution of participants above and below the zero line of the $x$-axis indicate that the worse stimulus discriminability was in the awareness test, the better was retrieval performance in the experiment. Hence, conscious stimulus discriminability might have counteracted unconscious paired-associative encoding and retrieval. (B) Experiment 2. Discrimination performance was regressed onto retrieval performance using the data from the subjects who had gained insight into the task principle during the initial suprathreshold version of experiment 2. The significant size of the $y$-axis intercept indicates that the retrieval effect persists when potential contributions of conscious stimulus discrimination are excluded.

\section{MATERIALS AND METHODS}

\section{Participants}

Thirty-six new participants (18 women and 18 men; age range: 20-34 years; $M=25.11, \mathrm{SD}=2.96)$ took part in experiment 2 for remuneration or course credit. Participants were normally sighted native German speakers, reported no current or past neurological or psychiatric disorders, and denied taking prescription or illegal drugs. Participants were kept naïve as to the purpose of the experiment (subliminal perception and memory). They were misinformed that the subliminal experimental versions were about attention (encoding part) and language (retrieval part).

\section{Design and procedure}

Design and procedure were identical to experiment 1 with three exceptions: (a) All participants performed the suprathreshold experimental version first, followed by two subliminal runs with (b) each a single encoding trial per word pair. (c) Interviews following the suprathreshold experiment assessed whether participants were aware of conceptual connections between encoding word pairs and analogs (e.g., that "table-car" was presented at encoding and the related word pair "desk-bus" at retrieval). We hypothesized that the insight into this conceptual connection between study and test word pairs was the relevant aspect in framing participants' taskset for the subsequent unconscious processing of subliminal word pairs.

\section{RESULTS AND DISCUSSION}

Fifteen of 36 participants had noticed the conceptual correspondence between at least one encoding word pair and one retrieval word pair (e.g., "table-car" followed by "desk-bus"). We considered these 15 participants to have gained insight into the task structure.

No participant reported to have suspected or perceived subliminal stimuli during the experiment. The group analysis of the data from the awareness test yielded a non-significant result $\left(M_{\text {correct }}=49.5 \% \mathrm{SD}_{\text {correct }}=7.1 \%\right), t(35)=-0.362, p=0.719$, $r^{2}=0.004$, and no participant reached more than 20 correct choices ( $=5 \%$-cut-off of the chance distribution) in the forcedchoice test.

The percentage of correct answers given in the attention task during the masking sequences of the experiment was high $(M=91.6 \%$; $\mathrm{SD}=9.3 \%)$. This high accuracy indicates that participants were visually fixating the screen center and were closely attending to subliminal stimulations.

We computed an ANOVA for the two runs with subliminal presentations using the percentage of fit answers as dependent variable and including the within-subjects factors Retrieval Condition (analogs versus broken analogs) and Run (first versus second run). The between-subjects factor was Insight (yes versus no). There was a significant main effect of Retrieval Condition with analogs receiving more fit answers $(M=35.7 \%$, $\mathrm{SD}=15.1 \%)$ than broken analogs $(M=32.9 \%, \mathrm{SD}=14.1 \%), F(1$, $34)=4.57, p=0.040, \eta_{p}^{2}=0.119$, which corroborated the finding of experiment 1 of a flexible expression of unconsciously acquired associations following a single encoding trial. This main effect was driven by the variable Insight, as indicated by the interaction of Retrieval Condition $\times$ Insight, $F(1,34)=5.84, p=0.021$, 
$\eta_{p}^{2}=0.147$ (Figure 3B). Post hoc $t$-tests revealed that participants who had gained insight into the conceptual correspondence between encoding word pairs and test word pairs during the initial suprathreshold run gave more fit answers to analogs $(M=40.8 \%$, $\mathrm{SD}=14.8 \%)$ than broken analogs $(M=33.5 \%, \mathrm{SD}=15.0 \%)$ during the subliminal runs $\left[t(14)=2.95, p=0.011, r^{2}=0.383\right]$. In contrast, subjects who gained no insight gave an equal number of fit answers to analogs $(M=31.9 \%, \mathrm{SD}=14.5 \%)$ and broken analogs $(M=32.4 \%, \mathrm{SD}=13.6 \%)$ during the subliminal runs $\left[t(20)=-0.218, p=0.830, r^{2}=0.002\right]$.

We computed a regression analysis using the performance on the awareness test (percentage of correct answers minus percentage of incorrect answers) as predictor and the performance achieved in the indirect retrieval test as criterion (percentage of fit answers to analogs minus percentage of fit answers to broken analogs; Greenwald et al., 1995). The group of participants who had gained insight into the relationship between encoding and retrieval word pairs during the initial suprathreshold version of the experiment and who had shown significant effects of subliminal perception yielded an $y$-axis intercept $(0.042, \mathrm{SE}=0.013)$ significantly greater than zero $[t(14)=3.151, p=0.008]$. This indicates that effects of subliminal perception persisted even if potential contributions of conscious stimulus discrimination were excluded. The slope $(B=-0.149, \mathrm{SE}=0.139, \beta=-0.286)$ was not significant $[t(14)=-1.075, p=0.302$; Figure $4 \mathbf{B}]$ suggesting that conscious stimulus discriminability as assessed in the awareness test was unrelated to implicit retrieval performance as measured in the experiment.

\section{GENERAL DISCUSSION}

We examined whether unconscious information processing exhibits computational characteristics of episodic memory, namely encoding in one single trial (McClelland et al., 1995; O'Reilly and Rudy, 2000, 2001; Norman and O'Reilly, 2003), new association formation (Squire, 1992; Cohen and Eichenbaum, 1993; Rolls and Treves, 1998; O'Reilly and Rudy, 2000, 2001; Norman and O'Reilly, 2003), and representational flexibility (Cohen and Eichenbaum, 1993; Frank et al., 2003). In both experiments, the subjective semantic distance between retrieval words was judged smaller if semantic neighbors of retrieval words had been presented subliminally side-by-side rather than combined with another word. Thus, even though retrieval words differed from encoding words, their mere analogy to concepts combined in encoding pairs decreased their perceived semantic distance. This result suggests a flexible expression of unconsciously acquired semantic word-word associations. One subliminal encoding trial was sufficient for participants to encode new semantic associations between subliminal words. There was no statistically significant increase in retrieval performance when encoding word pairs were presented in nine instead of one encoding trial. These findings indicate that unconscious associative encoding can be very rapid (McClelland et al., 1995; O'Reilly and Rudy, 2000, 2001; Norman and O'Reilly, 2003). Furthermore, retrieval effects in both experiments appeared following a study-test interval of 5 min. Hence, memories of subliminal words may outlast many minutes exceeding short-term and entering long-term memory storage (Shiffrin and Atkinson, 1969). These effects of subliminal stimulus processing depended on an initial understanding of the task structure.

A contamination of unconscious encoding by the conscious perception of subliminal words or fragments thereof is unlikely because the post-experimental interview and the awareness test confirmed the absence of a conscious access to subliminal words. Although the number of trials (30 trials) in the awareness test was rather small, we consider a conscious contamination of the experimental effects improbable because participants' discrimination performance in the awareness test was neither above chance level (Cheesman and Merikle, 1984) nor positively related to performance on the implicit retrieval test given in the experiment (Greenwald et al., 1995). Even if potential contributions by conscious stimulus perception existed, the experimental effects of encoding and retrieval persisted when such contributions were regressed out (Greenwald et al., 1995).

An interesting finding was that only those participants who had acquired some understanding of the task structure during an initial suprathreshold task version generated the reported effects of unconscious associative encoding and retrieval. Because the suprathreshold version contained other stimuli than those presented in the subliminal runs, the suprathreshold version did not prime subliminal words. Instead, the suprathreshold run allowed participants to gain insight into the conceptual analogy between encoding word pairs (table-car) and retrieval word pairs (deskbus). This insight into the task structure had apparently installed a task-set (Monsell, 2003) that was steering the processing of the subliminal word pairs (see also Martens and Kiefer, 2009). The necessity of installing a task-set by way of conscious processing for successful unconscious memory formation does not question the participants' unawareness of encoding stimuli and their unawareness of the formed memories that guided their choices at test. Although the abstract task principles were available to consciousness, the specific learning material and the formed memories were not. In fact, several studies revealed that the processing of subliminal information is under intentional control, rather than automatic (Naccache and Dehaene, 2001; Abrams et al., 2002; Kunde et al., 2003; Dehaene et al., 2006; Eckstein and Perrig, 2007; Kiesel et al., 2009; Martens and Kiefer, 2009; Kiefer and Martens, 2010). For example, Eckstein and Perrig (2007) found that the intention to classify the same words with respect to different semantic categories had a differential effect on measures of subliminal priming. Priming varied with the instructed semantic category in all experiments. Furthermore, a semantic versus perceptual induction task could activate a semantic or perceptual task-set and thereby modulate the sort of the subliminal priming effects (Martens and Kiefer, 2009; Kiefer and Martens, 2010). These and the current findings suggest that the processing of subliminal stimuli is facilitated by the previous built-up of specific expectations and the sensitization of the task-specific processing pathways. It appears that cognitive control can constrain both conscious and unconscious stimulus processing.

A crucial difference between the present experiments and other subliminal priming studies, such as those cited above (Kiesel et al., 2009; Martens and Kiefer, 2009; Kiefer and Martens, 2010), exists with respect to the nature of the formed memory representations and their duration over time. Subliminal semantic priming 
has mostly been accounted for in terms of short-lived prospective or preparatory processing initiated by the prime (Foster and Davis, 1984; Greenwald et al., 1996; Bowers, 2003; Kouider and Dehaene, 2007), such as spreading activation (Collins and Loftus, 1975). Spreading activation is short-lived and therefore cannot explain our subliminal effects that survived a study-test interval of several minutes in the current and past experiments (Henke et al., 2003; Duss et al., 2011). A retrospective account (Masson and Bodner, 2003) appears more appropriate for such findings because the retrospective account postulates that a prime establishes a memory trace through neuroplastic changes - e.g., induced by long-term potentiation - that benefit the later processing of the target. The long retention period brings our results into the realm of long-term memory and suggests this terminology ("encoding" and "retrieval") rather than the priming terminology ("prime" and "target").

The feasibility of rapid associative encoding and retrieval of word pairs without consciousness of retrieval has also been investigated with suprathreshold word presentations at both encoding and retrieval and with implicit (i.e., indirect) retrieval instructions. These studies included healthy participants and patients with amnesia. Graf and Schacter (1985) had amnesic patients and healthy control participants study pairs of unrelated words, such as "window-reason," in a single encoding trial. For retrieval, participants were presented with cues, such as "window-rea...," or new word combinations, such as "kitchen-rea..." (kitchen was combined with another word for study). Participants were required to produce the first word that came to mind as a completion of the word stem. The word stem was expected to be more often completed with the study word when old instead of recombined pairs were presented. This result would speak for an implicit retrieval of the word-word association. Indeed, controls and amnesic patients exhibited implicit memory for the studied word pairs following a semantic encoding of word pairs. Importantly, implicit memory for the new associations occurred independently of the level of explicit remembering. Similar implicit-explicit dissociations were found in memory-impaired patients of various etiologies using verification tasks such as identification of old versus recombined word pairs (Gabrieli et al., 1997), reading time for old versus recombined word pairs (Moscovitch et al., 1986), and lexical decision for old versus recombined word pairs (Goshen-Gottstein et al., 2000). When Schacter and Graf (1986) reanalyzed their original data (Graf and Schacter, 1985) and added new data, they found that the acquisition and unconscious retrieval of new word-word associations following semantic study was intact in mildly to moderately memory-impaired patients, but impaired in severely memory-impaired patients. Perhaps this and other results (Shimamura and Squire, 1989) can be explained by the dependence of unconscious association formation on the hippocampus

\section{REFERENCES}

Abrams, R. L., Klinger, M. R., and Greenwald, A. G. (2002). Subliminal words activate semantic categories (not automated motor responses). Psychon. Bull. Rev. 9, 100-106.

Bowers, J. S. (2003). "An abstractionist account of masked and long-term

and related structures. While conscious association formation might require the processing within a large hippocampal network, unconscious association formation might recruit a smaller neural population and therefore be intact in patients with mild amnesia due to only partial tissue damage. However, even for unconscious association formation a critical minimal amount of intact hippocampal tissue might be required for successful associative processing, which might not have been the case in severely memoryimpaired patients. In fact, evidence is accumulating that both conscious and unconscious rapid associative encoding and retrieval are impaired in severely amnesic patients with hippocampal damage. One-trial associative encoding and retrieval with different presentation formats at study and test requiring a flexible representation in memory was impaired in amnesic patients when encoding and retrieval were implicit, i.e., not directly instructed (Rajaram and Coslett, 2000a,b; Ryan et al., 2000; Verfaellie et al., 2006; Hannula et al., 2007). On the other hand, implicit single item encoding and retrieval remained intact in the same patients. This evidence suggests that processing features considered typical of episodic memory, namely a rapid associative encoding and a flexible retrieval, require hippocampal processing even if implicitly tested.

While the above results are suggesting that rapid associative encoding and flexible retrieval require hippocampal processing even if implicitly tested, our current results suggest that the same processing features are operational in healthy participants even if consciousness of both encoding and retrieval is securely excluded. Because speed of encoding, long-term retention, associativity, and representational flexibility are key features of episodic memory, operate with and without consciousness, and apparently require hippocampal processing, the question arises whether consciousness of encoding and retrieval is a necessary condition for episodic memory formation and expression (Henke, 2010). However, the where and when of happenings is an integral part of episodic memories (Tulving, 1985, 2001) and a firm claim of an unconscious form of episodic memory must await evidence of the unconscious formation of what-where-when memories (Tulving, 1985, 2001). Future research on unconscious association formation might benefit from excluding the contamination of unconscious by conscious processes when presenting the encoding material subliminally. Research on unconscious processing might also benefit from installing an appropriate task-set before experimentation. The use of neuroimaging methods will reveal to what extent neural networks underlying unconscious and conscious associative encoding/retrieval overlap.

\section{ACKNOWLEDGMENTS}

This research was supported by Swiss National Science Foundation grant 320000-114012. We thank Katharina Ledermann for her help with data collection.

Cohen, N. J., and Eichenbaum, H. (1993). Memory, Amnesia and the Hippocampal System. Cambridge: MIT Press.

Collins, A. M., and Loftus, E. F. (1975). A spreading-activation theory of semantic processing. Psychol. Rev. $82,407-428$.
Cook, N. J. (1986). The Brain Code London: Methuen Press.

Degonda, N., Mondadori, C. R. A. Bosshardt, S., Schmidt, C. F., Boesiger, P., Nitsch, R. M., Hock, C., and Henke, K. (2005). Implicit associative learning engages the hippocampus and interacts with explicit 
associative learning. Neuron 46, 505-520.

Dehaene, S., Changeux, J. P., Naccache, L., Sackur, J., and Sergent, C. (2006). Conscious, preconscious, and subliminal processing: a testable taxonomy. Trends Cogn. Sci. (Regul. Ed.) 10, 204-211.

Dunbar, K., and Blanchette, I. (2001). The in vivo/in vitro approach to cognition: the case of analogy. Trends Cogn. Sci. (Regul. Ed.) 5, 334-339.

Duss, S. B., Oggier, S., Reber, T. P., and Henke, K. (2011). Formation of semantic associations between subliminally presented face-word pairs. Conscious. Cogn. 20, 928-935.

Eckstein, D., and Perrig, W. J. (2007). The influence of intention on masked priming: a study with semantic classification of words. Cognition 104, 345-376.

Eichenbaum, H., Schoenbaum, G., Young, B., and Bunsey, M. (1996). Functional organization of the hippocampal memory system. Proc. Natl. Acad. Sci. U.S.A. 93, 13500-13507.

Foster, K. I., and Davis, C. (1984). Repetition priming and frequency attenuation in lexical access. J. Exp. Psychol. Learn. Mem. Cogn. 10, 680-698.

Frank, M. J., Rudy, J. W., and O'Reilly, R. C. (2003). Transitivity, flexibility, conjunctive representations, and the hippocampus. II. A computational analysis. Hippocampus 13, 341-354.

Gabrieli, J. D. E., Keane, M. M., Zarella, M. M., and Poldrack, R. A. (1997). Preservation of implicit memory for new associations in global amnesia. Psychol. Sci. 8, 326-329.

Goshen-Gottstein, Y., Moscovitch, M., and Melo, B. (2000). Intact implicit memory for newly formed verbal associations in amnesic patients following single study trials. Neuropsychology 14, 570-578.

Graf, P., and Schacter, D. L. (1985). Implicit and explicit memory for new associations in normal and amnesic subjects. J. Exp. Psychol. Learn. Mem. Cogn. 11, 501-518.

Greene, A. J. (2007). Human hippocampal-dependent tasks: is awareness necessary or sufficient? Hippocampus 7, 429-433.

Greenwald, A. G., Draine, S. C., and Abrams, R. L. (1996). Three cognitive markers of unconscious semantic activation. Science 273, 1699-1702.

Greenwald, A. G., Klinger, M. R., and Schuh, E. S. (1995). Activation by marginally perceptible ("subliminal”) stimuli: dissociation of unconscious from conscious cognition. J. Exp. Psychol. Gen. 124, 22-42.
Hannula, D. E., Ryan, J. D., Tranel, D., and Cohen, N. J. (2007). Rapid onset relational memory effects are evident in eye movement behavior, but not in hippocampal amnesia. J. Cogn. Neurosci. 19, 1690-1705.

Henke, K. (2010). Processing modes rather than consciousness distinguish between memory systems. Nat. Rev. Neurosci. 11, 523-532.

Henke, K., Buck, A., Weber, B., and Wieser, H. G. (1997). Human hippocampus establishes associations in memory. Hippocampus 7, 249-256.

Henke, K., Mondadori, C. R. A., Treyer, V., Nitsch, R. M., Buck, A., and Hock, C. (2003). Nonconscious formation and reactivation of semantic associations by way of the medial temporal lobe. Neuropsychologia 41, 863-876.

Henke, K., Weber, B., Kneifel, S., Wieser, H. G., and Buck, A. (1999). Human hippocampus associates information in memory. Proc. Natl. Acad. Sci. U.S.A. 96, 5884-5889.

Kiefer, M., and Martens, U. (2010). Attentional sensitization of unconscious cognition: task sets modulate subsequent masked semantic priming. J. Exp. Psychol. Gen. 139, 464-489.

Kiesel, A., Kunde, W., Pohl, C., Berner, M. P., and Hoffmann, J. (2009). Playing chess unconsciously. J. Exp. Psychol. Learn. Mem. Cogn. 35, 292-298.

Kouider, S., and Dehaene, S. (2007). Levels of processing during nonconscious perception: a critical review of visual masking. Philos. Trans. R. Soc. Lond. B Biol. Sci. 362, 857-875.

Kunde, W., Kiesel, A., and Hoffmann, J. (2003). Conscious control over the content of unconscious cognition. Cognition 88, 223-242.

Martens, U., and Kiefer, M. (2009). Specifying attentional top-down influences on subsequent unconscious semantic processing. $A d v$. Cogn. Psychol. 5, 56-68.

Masson, M. E. J., and Bodner, G. E. (2003). "A retrospective view of masked priming: toward a unified account of masked and long-term priming," in Masked Priming: State of the Art, eds S. Kinoshita and S. J. Lupker (New York: Psychology Press), 57-94.

Mayes, A., Montaldi, D., and Migo, E. (2007). Associative memory and the medial temporal lobes. Trends Cogn. Sci. (Regul. Ed.) 11, 126-135.

McClelland, J. L., McNaughton, B. L., and O'Reilly, R. C. (1995). Why there are complementary learningsystems in the hippocampus and neocortex - insights from the successes and failures of connectionist models of learning and memory. Psychol. Rev. 102, 419-457.

Monsell, S. (2003). Task switching. Trends Cogn. Sci. (Regul. Ed.) 7, 134-140.

Moscovitch, M., Winocur, G., and McLachlan, D. (1986). Memory as assessed by recognition and reading time in normal and memoryimpaired people with Alzheimer's disease and other neurological disorders. J. Exp. Psychol. Gen. 115, 331-347.

Naccache, L., and Dehaene, S. (2001). Unconscious semantic priming extends to novel unseen stimuli. Cognition 80, 215-229.

Norman, K. A., and O'Reilly, R. C. (2003). Modeling hippocampal and neocortical contributions to recognition memory: a complementarylearning-systems approach. Psychol. Rev. 110, 611-646.

O'Reilly, R. C., and Rudy, J. W. (2000). Computational principles of learning in the neocortex and hippocampus. Hippocampus 10, 389-397.

O'Reilly, R. C., and Rudy, J. W. (2001). Conjunctive representations in learning and memory: principles of cortical and hippocampal function. Psychol. Rev. 108, 311-345.

Rajaram, S., and Coslett, H. B. (2000a). Acquisition and transfer of new verbal information in amnesia: retrieval and neuroanatomical constraints. Neuropsychology 14 427-455.

Rajaram, S., and Coslett, H. B. (2000b). New conceptual associative learning in amnesia: a case study. J. Mem Lang. 43, 291-315.

Reber, P. J., Knowlton, B., and Squire, L. R. (1996). Dissociable properties of memory systems: differences in the flexibility of declarative and nondeclarative knowledge. Behav. Neurosci. 110, 861-871.

Reder, L. M., Park, H., and Kieffaber, P. D. (2009). Memory systems do not divide on consciousness: reinterpreting memory in terms of activation and binding. Psychol. Bull. 135 , 23-49.

Rolls, E. T., and Treves, A. (1998) Neural Networks and Brain Function. Oxford: Oxford University Press.

Ryan, J. D., Althoff, R. R., Whitlow, S. and Cohen, N. J. (2000). Amnesia is a deficit in relational memory. Psychol. Sci. 11, 454-461.

Schacter, D. L., and Graf, P. (1986). Preserved learning in amnesic patients: perspectives from research on direct priming. J. Clin. Exp. Neuropsychol. 8, 727-743.

Shiffrin, R. M., and Atkinson, R. C. (1969). Storage and retrieval processes in long-term memory. Psychol. Rev. 76, 179-193.

Shimamura, A. P., and Squire, L. R. (1989). Impaired priming of new associations in amnesia. J. Exp. Psychol. Learn. Mem. Cogn. 15, 721-728.

Silberman, Y., Miikkulainen, R., and Bentin, S. (2005). Associating unseen events - semantically mediated formation of episodic associations. Psychol. Sci. 16, 161-166.

Smith, C., and Squire, L. R. (2005). Declarative memory, awareness, and transitive inference. J. Neurosci. 25, 10138-10146.

Squire, L. R. (1992). Memory and the hippocampus - a synthesis from findings with rats, monkeys, and humans. Psychol. Rev. 99, 195-231.

Sutherland, R. J., and Rudy, J. W. (1989). Configural association theory - the role of the hippocampal-formation in learning, memory, and amnesia. Psychobiology 17, 129-144.

Treves, A., and Rolls, E. T. (1994). Computational analysis of the role of the hippocampus in memory. Hippocampus 4, 374-391.

Tulving, E. (1985). Memory and consciousness. Can. Psychol. 26, 1-12.

Tulving, E. (2001). Episodic memory and common sense: how far apart? Philos. Trans. R. Soc. Lond. B Biol. Sci. 356, 1505-1515.

Verfaellie, M., Martin, E., Page, K., and Keane, M. M. (2006). Implicit memory for novel conceptual associations in amnesia. Cogn. Affect. Behav. Neurosci. 6, 91-101.

Conflict of Interest Statement: The authors declare that the research was conducted in the absence of any commercial or financial relationships that could be construed as a potential conflict of interest.

Received: 05 July 2011; paper pending published: 30 August 2011; accepted: 02 November 2011; published online: 22 November 2011.

Citation: Reber TP and Henke K (2011) Rapid formation and flexible expression of memories of subliminal word pairs. Front. Psychology 2:343. doi: 10.3389/fpsyg.2011.00343

This article was submitted to Frontiers in Consciousness Research, a specialty of Frontiers in Psychology.

Copyright () 2011 Reber and Henke. This is an open-access article subject to a nonexclusive license between the authors and Frontiers Media SA, which permits use, distribution and reproduction in other forums, provided the original authors and source are credited and other Frontiers conditions are complied with. 


\section{APPENDIX \\ POST-EXPERIMENTAL INTERVIEW}

Remember the two parts of the experiment in which you saw black and white dot patterns and you performed the attention task. Did you notice anything else than the black and white dot patterns or did you notice any regularity within these dot patterns?

\section{If participant answers "yes" to the above question:}

Describe what you saw:

Did you see it clearly?

How big was it?

Where on the screen did you see it?

Did you see this always or just sometimes?

In case you saw it only sporadically, when and how often did you see it?

\section{Questions and information for all participants:}

Did you potentially see contours of letters or the contour of a word?

Words were presented very briefly - for $17 \mathrm{~ms}$ - between the black and white dot patterns. Now that you know this, do you think that you sometimes had a hunch of letters or words?

If yes, where exactly did you see the letters or words? How large were they?

In fact, we presented words to the left and right of the fixation cross and they spanned nearly the full width of the screen. Now that you know all this, do you think you could see any aspect of words? 